\title{
TTR
}

Traduction, terminologie, rédaction

\section{Baudelaire, l'opium et la poésie : digestion, digression et adaptation}

\section{Camille Muris-Prime}

Volume 27, numéro 1, 1er semestre 2014

URI : https://id.erudit.org/iderudit/1037124ar

DOI : https://doi.org/10.7202/1037124ar

Aller au sommaire du numéro

\section{Éditeur(s)}

Association canadienne de traductologie

ISSN

0835-8443 (imprimé)

1708-2188 (numérique)

Découvrir la revue

Citer cet article

Muris-Prime, C. (2014). Baudelaire, l'opium et la poésie : digestion, digression et adaptation. TTR, 27(1), 213-227. https://doi.org/10.7202/1037124ar

\section{Résumé de l'article}

Baudelaire n'est pas que le traducteur de Poe. Il a aussi adapté Confessions of an English Opium Eater de Thomas De Quincey. Devenu Un mangeur d'opium et placé en deuxième partie des Paradis artificiels, ce texte est utilisé par Baudelaire de plusieurs façons. Le poète s'attache à remanier le texte de sorte à faire du travail de traduction un véritable travail d'adaptation, et il le place aussi au sein d'un diptyque afin de l'utiliser dans sa démonstration sur les drogues. L'analyse du texte de Baudelaire permet de comprendre la façon dont se développe son projet esthétique qui consiste à comparer l'état dans lequel met la drogue au paradis poétique auquel tente d'accéder le poète. La traduction permet aussi à Baudelaire de préciser sa position éthique puisqu'il réussit à adapter l'oeuvre de De Quincey tout en la laissant visible, la rendant lisible tout en dialoguant avec elle. Si Un mangeur d'opium est un texte assez peu connu, il est pourtant un laboratoire où la traduction permet à Baudelaire de mettre en abyme sa propre pratique poétique. 


\title{
Baudelaire, l'opium et la poésie: digestion, digression et adaptation
}

\author{
Camille Muris-Prime \\ University College London
}

\section{Résumé}

Baudelaire nest pas que le traducteur de Poe. Il a aussi adapté Confessions of an English Opium Eater de Thomas De Quincey. Devenu Un mangeur d'opium et placé en deuxième partie des Paradis artificiels, ce texte est utilisé par Baudelaire de plusieurs façons. Le poète s'attache à remanier le texte de sorte à faire du travail de traduction un véritable travail d'adaptation, et il le place aussi au sein d'un diptyque afin de l'utiliser dans sa démonstration sur les drogues. L'analyse du texte de Baudelaire permet de comprendre la façon dont se développe son projet esthétique qui consiste à comparer l'état dans lequel met la drogue au paradis poétique auquel tente d'accéder le poète. La traduction permet aussi à Baudelaire de préciser sa position éthique puisqu'il réussit à adapter l'ouvre de De Quincey tout en la laissant visible, la rendant lisible tout en dialoguant avec elle. Si Un mangeur d'opium est un texte assez peu connu, il est pourtant un laboratoire où la traduction permet à Baudelaire de mettre en abyme sa propre pratique poétique.

Mots-clés: Baudelaire, De Quincey, poésie, adaptation, traduction, dialogue, esthétique moderne

\begin{abstract}
Baudelaire did not only translate Poe. He also translated De Quincey's Confessions of an English Opium Eater. Un mangeur d'opium was published in the second part of Baudelaire's Paradis artificiels, and Baudelaire used it in different ways. He reworked the text with the goal of adapting rather than translating - it was a key element of this diptych, used to examine the effects of drugs. Baudelaire's textual analysis provides a better understanding of his aesthetic project, which was to compare the narcotic experience to the poet's paradise. Baudelaire was thus able to use his translation to outline his ethical position by adapting De Quincey's work, leaving it visible-and making it readable-all the while taking part in a dialogue with the text. Though Un mangeur d'opium is not a famous text, it is nonetheless a testing ground for Baudelaire's "mise en abyme" for his own poetic practice.
\end{abstract}

Keywords: Baudelaire, De Quincey, poetry, adaptation, translation, dialogue, modern aesthetics 
C'est là [Les paradis artificiels] non le message adressé par un drogué à des drogués, mais le livre écrit par un poète pour tous ceux qui considèrent que la seule vraie drogue, la drogue absolue, est la Poésie, et le seul problème, sa naissance et sa connaissance. (Pichois, 1975, p. 1)

De 1857 à 1860, et plus précisément en 1858 et 1859, Baudelaire traduit, commente, découpe et finalement adapte Confessions of an English Opium Eater de Thomas De Quincey. Cette adaptation, destinée à la Revue contemporaine d'Alphonse de Calonne et qui a valu à son artisan poète de grandes souffrances, trouve sa place de façon concomitante sous le titre "Un mangeur d'opium» au sein de la seconde œuvre publiée par Baudelaire de son vivant: Les paradis artificiels.

Si l'on parle d'adaptation - « [a]nalyse, non traduction, il faut y insister» dit Claude Pichois (1975, p. 1363) - c'est parce que Baudelaire fait beaucoup plus que «traduire», au sens technique, la prose de De Quincey. Sous la contrainte de son exigeant éditeur, puis parce que le "Mangeur d'opium» est destiné à constituer la seconde partie d'une œuvre, il la réorganise, la commente, la met en scène, s'y met en scène en lecteur et grand manitou narrateur, et surtout la coupe, la digère, la fait sienne, la réoriente. Baudelaire lui-même affirme, dans une lettre à Poulet-Malassis datée du 16 février 1860, ne pas avoir seulement traduit:

De Quincey est un auteur affreusement conversationniste et digressionniste, et ce n'était pas une petite affaire que de donner à ce résumé une forme dramatique et d'y introduire l'ordre. De plus il s'agissait de fondre mes sensations personnelles avec les opinions de l'auteur original et d'en faire un amalgame dont les parties fussent indiscernables. Ai-je réussi? (1973a, p. 660)

Ce qui constitue l'intérêt de la relation de Baudelaire avec De Quincey et de la notion d'adaptation est le fait qu'au sein du Mangeur d'opium s'élaborent deux paroles. Celle de Baudelaire est seconde par rapport à celle de De Quincey et elle ne vaut qu'au sein de la première, elle n'existe que grâce au matériau du texte qu'elle traduit. Elle n'atteint la plénitude de son propos que parce qu'elle est dialogue, parce qu'elle est traversée par et de cette expérience du monde et du langage qui est celle de De Quincey. Or, cette adaptation de la parole d'autrui, et d'une parole qui est 
autobiographique, permet à Baudelaire de construire une véritable esthétique, puisqu'Un mangeur d'opium prend place au sein des Paradis artificiels, œuvre constituée par le poète lui-même.

Un mangeur d'opium se construit donc dans le dialogue de plusieurs façons: tout d'abord dans sa forme même, puis parce qu'il est construit comme le diptyque d'un autre texte, et que grâce à la médiation, à la rencontre et la traduction de la parole d'autrui, il met en place une esthétique nouvelle et originale. C'est donc grâce et au sein de la parole d'autrui que se construisent le texte et l'esthétique baudelairiens. La suite du présent article consiste à analyser les différentes formes que prend ce dialogue, et cherche à montrer que la traduction a permis à Baudelaire d'élaborer une parole originale.

\section{1.}

Que fait donc Charles Baudelaire du texte de Thomas De Quincey? Selon ses dires, Un mangeur d'opium serait un amalgame, c'est-àdire un texte constitué de matériaux textuels variés. Nous sommes donc face à un texte à la composition originale. Essayer de le faire entrer dans une case générique relève du casse-tête littéraire, car l'on se rend vite compte qu'il ne s'agit nullement de la «simple» traduction d'un récit autobiographique, mais plutôt d'un millefeuilles énonciatif.

Thomas De Quincey est désigné dès le premier chapitre, dans l'œuvre même et par le narrateur, comme l'«auteur». L'auteur du livre que l'on est en train de lire n'est donc pas celui qui parle et que l'on rencontre dès le premier paragraphe. Ce stratagème permet à plusieurs voix de s'installer au sein du texte du Mangeur d'opium, voix qui vont prendre la parole à tour de rôle. On y entend plusieurs énonciateurs, plusieurs $j e$, plusieurs textes: on y trouve par exemple De Quincey narrateur, ou bien d'autres je qui sont autant de postures énonciatrices prises par Baudelaire. Le montage de ces différents éléments se fait de manière subtile de façon à constituer un tout cohérent. Il faut analyser la totalité du premier chapitre, les «Précautions oratoires», pour se rendre compte d'une façon plus précise du travail de stratification de textes et de voix auquel se livre Baudelaire, et qui est caractéristique du reste de l'œuvre.

Le premier chapitre se développe ainsi en six paragraphes qui expriment les différents modes énonciatifs utilisés par le poète 
français. Le premier paragraphe commence par la traduction d'une partie du texte de De Quincey, la prière où l'opium est personnifié et magnifié, laquelle ne se situe nullement au seuil de l'œuvre anglaise ${ }^{1}$. Charles Baudelaire l'a en effet placée ici afin de donner le ton et la gamme de l'œuvre et constituer les "premières mesures de l'orchestre $»^{2}$, comparant ainsi son travail de traduction à l'adaptation musicale. Cette traduction se présente comme une citation puisqu'elle est placée entre guillemets. Nous nous trouvons ici face à un premier type de recomposition (par déplacement). Puis, dans la seconde partie de ce même paragraphe, Baudelaire reprend la parole, et met à distance celle de celui qui va être désigné comme l'«auteur" par un narrateur critique et commentateur; ce qui constitue un second type de recomposition.

Après avoir de nouveau laissé la parole à De Quincey dans le second paragraphe, où se déploie donc un je qui appartient au narrateur autobiographique anglais, Baudelaire reprend la plume pour se faire rapporteur dans le troisième paragraphe, paraphrasant et résumant le texte anglais, ce qui constitue un troisième type de recomposition. L«auteur» est renvoyé à la troisième personne du singulier. Il arrive également à Baudelaire de couper, sans le signaler, des éléments du texte de De Quincey tout en gardant l'essentiel, ou d'ajouter quelques mots au sein de la prose de l'auteur anglais, ce qui a pour effet d'orienter le projet initial.

Tous ces éléments, les différentes postures et activités de Baudelaire sur le texte de De Quincey et autour de celui-ci, nous ont conduit à parler non seulement de traduction, mais aussi d'adaptation: le travail de Baudelaire consistant principalement à garder les grandes lignes du texte anglais tout en le réduisant, le résumant, le modifiant, le faisant sien. Baudelaire semble justifier cette entreprise en affirmant qu'il fallait «introduire de l'ordre» dans le texte anglais (1973a, p. 660). Il fait donc subir aux Confessions trois types de modifications: la suppression (il faut "abréger»), l'addetim (de simples syntagmes ou de paragraphes entiers) et la réorganisation.

1. V. De Quincey (1998 [1821]) et (2000 [1821]).

2. V. lettre du 5 janvier 1860: «J'ai enfin trouvé le début qui ressemble, par sa solennité, aux premières mesures d'un orchestre.» (Baudelaire, 1973a, p. 650-651) 
La distinction entre adaptation et traduction n'est pas toujours facile à établir. Il peut être problématique de vouloir distinguer absolument les deux activités $^{3}$, le risque étant de n'assigner la traduction qu'au passage le plus littéral possible d'un texte d'une langue à une autre. Ce faisant, la traduction ne serait plus alors traitée que comme une activité ancillaire par rapport au texte original, puisque toute intervention du traducteur sur ce dernier renverrait le texte traduit à n'être qu'une «adaptation". Il y a cependant un pas entre ce que Georges Bastin appelle «adaptation ponctuelle» et l'«adaptation globale» (1993, p. 476). La première relève de tout travail de traducteur et consiste à ajouter, supprimer ou modifier un mot, une expression ou une image de façon ponctuelle lors de la traduction, de façon à conserver la cohérence du discours, quitte à mettre en place des stratégies de compensation au sein du texte ${ }^{4}$. L'«adaptation globale» a, elle, pour ambition de réaliser «la visée de l'auteur» (ibid.) et considère donc le texte original comme le matériau (brut) de la construction à laquelle va se livrer l'adaptateur. Cette forme d'adaptation a pour caractéristique principale, à la différence de l'adaptation ponctuelle, d'affecter l'ensemble du texte d'arrivée (ibid., p. 478).

C'est à cette seconde forme d'adaptation que nous avons décidé de nous référer lorsque nous parlons d'adaptation du texte de De Quincey. Le travail de Baudelaire dépasse en effet largement le cadre de l'adaptation ponctuelle puisqu'il coupe, ajoute, déplace et réécrit de nombreux passages. À partir du texte anglais, Baudelaire réalise dans Un mangeur d'opium trois des quatre étapes de ce que M.A. Johnson appelle les «creative processes of adaptation » (1984,p. 423), à savoir l'ingestion (lecture de l'œuvre, collecte des données), la digestion (assimilation du texte - laquelle passe, entre autres, par la traduction dans le cas du Mangeur d'opium), et la réjection («editing, restyling, discarding redundancies, spotting weaknesses, eliminating, substituting with a view to making the work a masterpiece» (ibid., p. 422)). Le fait que Baudelaire traduise a donc son importance, mais celleci n'est qu'une étape de son travail sur le texte traduit. Car en le manipulant ainsi, il l'utilise au service à la fois d'un projet et d'un propos: celui de faire du Mangeur d'opium la seconde partie d'un

3. V. Gambier (1992).

4. V. Scott (2006). 
livre diptyque, et celui de démontrer la supériorité esthétique et métaphysique de la Poésie sur la drogue. La «mise en ordre» du texte de De Quincey, plus qu'une mise au pas, est ainsi avant tout «une mise en œuvre». Baudelaire ne fait pas que modifier le texte de De Quincey, il produit un texte qui est bien différent de son original et n'est pas qu'un "résumé». Baudelaire oriente son texte, au-delà de l'adaptation d'une autobiographie, vers une œuvre originale, qui désigne cependant toujours, de même qu'elle intègre en son sein, l'œuvre originelle: "Il s'agissait de fondre mes sensations personnelles avec les opinions de l'auteur original et d'en faire un amalgame» (Baudelaire, 1973a, p. 669). Ceci est montré par la présence d'un narrateur homodiégétique qui s'affiche. Le poète devient donc auteur du texte en français, presque au même titre que De Quincey l'est du texte en anglais.

Ainsi, avec Un mangeur d'opium, on pourrait presque donner une valeur générique à l'adaptation. Cela dépasse le cadre de la nature du texte, et c'est la marque de l'originalité de Baudelaire sur le plan formel. L'adaptation est presque élevée au rang de genre, car elle intègre tous les discours, rend compte d'une réalité à la fois formelle et esthétique, et fait du texte un morceau à deux voix où la seconde, celle du poète français, enveloppe et interprète la première. À la lecture du Mangeur d'opium, Victor Hugo s'est d'ailleurs exclamé : «Analyser ainsi, c'est créer!» (Pichois, 1967, p. 192). On peut en effet dire que Baudelaire crée une œuvre qui n'a pas son pareil en littérature, puisque sa caractéristique formelle est de donner à sa méthode de composition une valeur générique, un cadre qui va permettre au message du poète de s'épanouir, tout en respectant la parole de De Quincey et en communiquant l'expérience esthétique qu'elle révèle. Un mangeur d'opium, œuvre originale, est donc un texte qui inscrit au fond de sa pratique le dialogue et qui met en scène la discussion entre l'auteur et son traducteur.

\section{2.}

Le dialogue des voix au cœur du Mangeur d'opium est comme mis en abyme par la construction en deux parties des Paradis artificiels. Un mangeur d'opium y succède en effet au Poème du hachisch et vient donc lui répondre et dialoguer avec lui. Le poème du hachisch expose une théorie morale et esthétique dont Un mangeur d'opium 
va être la démonstration, l'exemple. C'est pourquoi il faut nous approcher à présent de la première partie des Paradis artificiels.

Dans Le poème du hachisch, Baudelaire expose tout d'abord une morale, qui aboutit à une condamnation de la drogue. Puis il crée un personnage fictif qui goûte à la drogue et semble accéder au bonheur. Grâce à la traduction insérée, Un mangeur d'opium va apporter une illustration de la condamnation du fait des "châtiments» subis par l'auteur anglais.

Si la drogue est condamnée dans Le poème du hachisch, c'est parce qu'elle est pour le poète le signe d'une «dépravation du goût de l'infini» (Baudelaire, 1976, p. 465). Elle représente une transgression: celle de l'homme qui veut se faire dieu ou tout du moins le remplacer, s'accorder une félicité d'un seul coup de dés: "Comment nommerons-nous l'homme qui veut acheter, avec un peu de monnaie, le bonheur et le génie?»(ibid.). Baudelaire reproche au hachisch le fait que sa consommation procure les illusions de la facilité créatrice et du bonheur, alors qu'elles ne sont que l'antichambre des cauchemars. Max Milner identifie deux raisons à la condamnation du hachisch par Baudelaire: "le sentiment d'innocence, de perfection et de toute puissance qu'il donne à l'homme, se faisant ainsi l'égal de Dieu» (1996, p. 21) et "l'infaillibilité avec laquelle il lui permet d'accéder à un état de béatitude qui devrait être le fruit de l'effort, la récompense de la vertu» (ibid.) - abstraction faite de l'ironie, naturellement... La seconde raison est plus importante selon Max Milner, car alors disparaît le sentiment de culpabilité, lequel est chez Baudelaire «la condition première de toute existence morale, l'aiguillon qui empêche l'être humain de se satisfaire d'un monde naturel irrémédiablement condamné» et qui permet donc à la Poésie de se réaliser. Si le hachisch est diabolique, c'est parce qu'il «fait croire celui qui s'y livre à la bonté naturelle de l'homme, et avant tout à sa propre bonté» (Milner, 2000, p. 112). Or, la nature humaine n'est pas «bonne» pour Baudelaire, d'où sa valorisation de l'artifice. La condamnation de la drogue s'inscrit donc dans l'esthétique baudelairienne de l'effort et de la quête «terrestre» du paradis, de ce monde idéal peu chrétien, de nature esthétique.

Cependant, si une qualité est reconnue à la drogue, c'est celle de laisser apercevoir à l'homme - entendu comme poète cet espace idéal qui doit être atteint par le travail poétique. Cette 
expérience est développée dans le chapitre «L'Homme-Dieu» du Poème du hachisch. Selon le poète, la drogue permet de modifier la perception et la sensibilité de façon équivalente à ce que cherche à atteindre Baudelaire dans ses professions de foi esthétique: exaltation du sens de la couleur et surtout mise en lumière des correspondances et des analogies, révélation de la possibilité d'une unité du monde. Perceptions synesthésiques, «accroissement monstrueux du temps et de l'espace», révélation de la "profondeur de la vie», et capacité de donner au langage un "pouvoir littéralement évocatoire» (ibid., p. 141), tout cela laisse à penser que l'expérience, ou l'expérience narrée, du hachisch a eu pour Baudelaire un rôle essentiel, puisquelle a offert au poète un moyen d'imaginer ce que serait l'état poétique idéal. Mais cela résonne de façon ambiguë avec la condamnation précédente du hachisch, de façon à troubler la bonne réception de l'œuvre, si l'on se place sur un plan moral. Il faut en effet aussi envisager Les paradis artificiels sous l'angle de la théorie esthétique, puisque, comme le souligne Michel Butor, c'est la comparaison avec l'état de drogué qui a permis à Baudelaire de donner une sorte de "mode d'emploi » de sa poésie (1992, p. 9). Le rapport du poète avec la drogue en devient esthétique, et le «rôle très précis » assigné alors aux Paradis artificiels est d'être l'"ouvrage fondamental [de Baudelaire] sur la nature de la poésie» (ibid., p. 10). Son ambition n'est pas de faire le catalogue des états dans lequel le poète peut se trouver lorsqu'il fait usage des psychotropes, mais plutôt de fournir à Baudelaire deux images qu'il pourra comparer et opposer à la poésie et à ses effets, que l'on pourrait appeler l'«état de poésie».

Un mangeur d'opium, qui expose et commente le «cas» de De Quincey, illustre cette exposition théorique qu'a livrée Baudelaire dans la première partie de l'œuvre. Les deux parties des Paradis artificiels fonctionnent en miroir. Dans chacune d'entre elles, la drogue - hachisch puis opium - est d'abord louée pour être ensuite condamnée:

L'ouvrage $[\ldots]$ est formé de deux tableaux confrontés [...], chacun comportant un éloge de la drogue, puis une condamnation de celle-ci, mais attention, celle-ci n'est pas condamnée selon les règles de la morale bourgeoise, elle est condamnée, elle est vaincue par la poésie qui est plus forte qu'elle. (Butor, 1992, p. 14) 
Dans Les confessions, la condamnation se trouve dans les cauchemars de De Quincey, ces moments où les voluptés de l'opium se transforment en tortures. Or, c'est en écrivant ses Confessions que De Quincey se libère de l'empire de la drogue. Baudelaire, lui, va tirer les conclusions de cet effort. En faisant l'adaptation du texte de De Quincey de façon à ce qu'il fonctionne comme l'illustration à la fois concrète et littéraire de ce qui était exposé dans Le poème du hachisch, il réalise aussi son projet esthétique, lequel consiste à faire du paradis atteint par la drogue une image du paradis poétique.

\section{3.}

Cette création d'une parole originale autour d'une parole exogène a plusieurs conséquences. Il y a d'abord la question de la fraternité: Baudelaire insiste ou montre, au long de son œuvre, les points communs qui le lient à l'auteur anglais. C'est le premier aspect du dialogue qui s'instaure entre les deux paroles. Lorsque l'on parle de «fraternité», on peut se placer sur deux registres. Le premier est biographique, mais plus relativement, psychologique. Il est possible de tracer un parallèle entre l'histoire personnelle de Thomas De Quincey et celle de Charles Baudelaire, lequel semble s'être reconnu dans l'image que donnait de lui l'auteur anglais. Ainsi, lui aussi a ressenti ce fort sentiment de sa particularité dès l'enfance (Baudelaire, 1975, p. 680); lui aussi fut élevé dans sa petite enfance au sein d'un cercle féminin composé de sa mère et de sa bonne, ce qu'il définit comme "mundus muliebris» (Baudelaire, 1973b, p. 30-31), et qui est selon lui responsable d'une certaine sensibilité qu'il retrouve chez l'auteur anglais; lui aussi fut «fertilisé par la douleur", par la perte symbolique de sa mère lorsqu'elle se remarie avec le beau-père honni, puis par la maladie vénérienne qu'il contracte jeune homme, et par la misère et les problèmes financiers qui furent son environnement toute sa vie adulte durant; et enfin, lui aussi eut recours à l'opium à des fins thérapeutiques et souffrit de procrastination.

Mais la fraternité qui lie les deux auteurs se noue principalement sur le plan esthétique. Si Baudelaire découvre en Thomas De Quincey un frère, c'est pour les thèmes que celui-ci développe et qui lui sont chers; c'est pour la beauté, prise dans son sens actif comme un objet qui suscite la réflexion, de sa prose et ses attitudes esthétiques. De Quincey ressemble à ce portrait que 
Baudelaire fait du représentant du Beau dans Les fusées et qui est l'expression de la mélancolie:

J'ai trouvé la définition du Beau, - de mon Beau. C'est quelque chose d'ardent et de triste, quelque chose d'un peu vague, laissant carrière à la conjecture $[. .$.$] . Le mystère,$ le regret aussi sont des caractères du Beau [...]. Quelque chose d'ardent et de triste, [...] et enfin, (pour que j'aie le courage d'avouer jusqu'à quel point je me sens moderne en esthétique), le Malheur [...]. Je ne conçois guère [...] un type de Beauté où il n'y ait du Malheur [...] on conçoit qu'il me serait difficile de ne pas conclure que le plus parfait type de Beauté virile est Satan, - à la manière de Milton. (1973a, p. 657-658).

La figure allégorisée de De Quincey-poète ressemble à ce Satan miltonien et fait de lui une sorte d'ange déchu, capable d'avoir atteint le sublime, ou tout du moins aperçu, et marqué par la douleur de la perte, de la chute, de la confrontation avec sa finitude humaine. Baudelaire fait de De Quincey une figure de la Beauté moderne et dépasse son œuvre, dans cette perspective. La Beauté moderne se réalise dans l'impossible dépassement de la finitude de la condition humaine et dans la conscience de celle$\mathrm{ci}^{5}$. Cela n'est possible que parce qu'il reconnaît une communauté de thèmes avec l'écrivain anglais, qu'il part du même constat, de la même quête vers l'Idéal esthétique, qui est dénué de toute moralité, dans le sens où la morale, qui est consécutive de la liberté humaine à choisir, est la marque de sa finitude, le choix révélant l'impossible connaissance absolue. Il y a donc entre les deux auteurs une fraternité qui n'est pas identité. C'est dans cette différence que va se déployer l'esthétique baudelairienne, son style.

Car en modifiant Les confessions, Baudelaire expose, puis refuse l'esthétique romantique de l'œuvre anglaise. C'est dans l'écriture même que les deux discours se lient et se séparent. La question esthétique est en effet au cœur de l'adaptation baudelairienne, tout comme elle l'est dans le texte de De Quincey, ainsi que l'affirme Éric Dayre: «De Quincey vise à retrancher la confession de la morale, pour la faire passer intégralement du côté de l'art» (2003, p. 184). Tous deux prêtent à l'opium - ou, pour Baudelaire, aux stupéfiants d'une façon plus générale - la

5. V. Les phares, Baudelaire, 1975, p. 13. 
capacité de faire croire au sujet qu'il est libéré de tout le poids de sa condition humaine (finitude, solitude, culpabilité et douleur), en lui donnant à voir l'objet esthétique absolu et «amoral», qui se situe hors du champ de la morale. La quête esthétique consiste à tenter, dans l'écriture, de recréer cet objet. Or, l'expérience du Mangeur d'opium montre que l'opium n'est producteur que d'illusions. La drogue, un temps libératrice du carcan humain, ne fait que rappeler l'homme à sa condition essentielle, et de manière violente, par l'empoisonnement, la dépendance, ce qui se caractérise par de violentes angoisses. Elle ramène l'homme à sa condition d'être moral et coupable. Baudelaire nomme ce rappel à l'ordre «châtiment» (1975, p. 480), châtiment de l'homme qui a voulu et a cru pouvoir être dieu, ou être capable de parler d'égal à égal avec lui.

Cependant, avec la drogue s'est ouvert pour l'homme un espace infini, où règne l'unité absolue, qui constitue une sorte de monde intelligible. Le Poète, celui qui est marqué par la Douleur, c'est-à-dire la conscience de la Chute et de la finitude humaine, la conscience de l'impossible accession à ce "paradis", le Poète donc, n'aura de cesse de retrouver ce "paradis perdu", de tenter de le re-nommer, au sens biblique de re-créer. Cette attitude est celle du Poète romantique, lequel est en quête d'un Absolu et est un être souffrant, car cet Absolu lui échappe sans cesse, et sa quête le renvoie toujours à sa condition humaine. Cette quête, qui se sait impossible, s'exprime chez De Quincey dans les digressions qui sont la marque de l'éternelle et infinie tentative de créer, nommer, trouver par le langage cet objet esthétique amoral.

Or, Baudelaire, tout en reconnaissant leur importance, supprime ces digressions, tout comme il retire à l'auteur sa parole confessante. Cela lui permet de "sauver» la quête esthétique de De Quincey. Il vient désamorcer l'échec programmé de la position quinceyenne en réduisant le texte aux visions de lopium et au châtiment, et il la renvoie à sa plus simple expression, c'est-àdire la culpabilité suscitée par l'incapacité à n'avoir pu surmonter la dépendance de la drogue ou de la religion, dépendance dont l'écriture porte comme la marque. Il allégorise cette quête nécessairement infructueuse en en faisant la marque de la Beauté. Cette Beauté est nécessairement «moderne». La quête elle-même devient l'objet esthétique et se réalise dans la Poésie. Baudelaire 
offre donc à l'homme la possibilité de se réaliser sans renier sa condition. Si la Beauté est moderne, faite de finitude, de " mouvant » et de "changeant", ainsi que d'aspiration à un éternel immuable, elle peut se réaliser dans le langage, dans la Poésie. Cela n'est rendu possible que parce qu'il avait auparavant montré dans Le poème $d u$ hachisch que le "paradis", lequel n'a rien de théologique, peut être atteint par le travail artistique. Il donne ainsi un sens à la douleur: elle est ce qui permet de créer et rend le sujet capable de réaliser le Beau.

Un mangeur d'opium permet donc à Baudelaire, en s'écartant de De Quincey, de développer sa théorie esthétique, ainsi que de la mettre en pratique. Car en utilisant le texte anglais il fait surgir cette Beauté, par la mise en abyme de la parole de De Quincey et de la description de ses visions qui révèlent la capacité évocatoire, poétique, du langage. En nous donnant à lire, en passant par la médiation de l'écriture d'autrui, il nous montre ce «paradis» qui se réalise et s'inscrit dans l'écriture des visions de Thomas De Quincey, dans leur réécriture. Il montre la capacité du langage, non pas à nommer de façon biblique, mais à évoquer. Chez De Quincey l'écriture comme l'opium renvoyaient l'homme à sa condition insurmontable. Chez Baudelaire la situation est différente, et cela est rendu possible grâce au montage des voix et de l'adaptation. En effet, l'écriture se situe au-dessus des visions libérées de l'emprise de l'opium. Mais parce qu'elle est capable d'évoquer ces visions, elle permet aussi la création d'un objet esthétique. Et celui-ci, même marqué par l'impossibilité de nommer l'Absolu, est alors cependant capable d'en rendre compte, bien que de façon incomplète, limitée, humaine.

La différence est donc fondamentale entre le projet de De Quincey et celui de Baudelaire. Il semble que seul le «moderne» puisse trouver sa réalisation: au terme d'un compromis, certes, celui de l'acceptation de la condition humaine qui est finitude; mais aussi conscience de l'infini, donc douloureuse. Chez Baudelaire, c'est au sein de la littérature, au sein de la "grammaire» que peut s'épanouir la Beauté. Et la drogue devient un prétexte à son surgissement. Le texte de De Quincey se fait alors à la fois exemple de celui-ci et de la capacité de la création poétique. C'est en tout cas ce que Baudelaire montre en déclarant dans $U n$ mangeur d'opium: 
Le prologue est fini, et je puis promettre au lecteur, sans crainte de mentir, que le rideau ne se relèvera que sur la plus étonnante, la plus compliquée et la plus splendide vision qu'ait jamais allumée sur la neige du papier la plume fragile du littérateur. (1975, p. 464).

C'est l'écriture qui est apte à révéler.

C'est donc via la littérature et l'expérience littéraire d'autrui que Baudelaire construit son esthétique; c'est par la découverte d'une fraternité avec l'originalité d'une parole qu'il révèle et illustre sa propre originalité, sa position esthétique qui devient une position éthique. Cette position, moderne, lui permet de créer un objet esthétique qui reste communicable à autrui, essentiellement "prostituable»; ce qui n'est pas le cas de l'objet esthétique "romantique», qui, dépassant l'homme, ne serait intelligible que pour Dieu. Baudelaire développe une théorie esthétique qui montre la conscience de l'homme, de sa finitude et de son incapacité essentielle à trouver le langage, un langage unique pour exprimer la douleur de cette condition. Sa théorie permet à l'homme d'atteindre à un paradis esthétique, celui des Poètes et des Esthètes, qui se réalise dans l'Art et qui permet au poète avec son lecteur d'échapper provisoirement à la solitude en renouant avec la communauté des vivants et des souffrants. Utiliser le texte de De Quincey est la marque de cette position esthétique qui devient une position éthique et place le Poète au sein du monde.

L'expérience de la traduction semble chez Baudelaire être secondaire, puisqu'elle est aussi mise à distance de l'auteur et commentaire du traducteur sur le texte traduit. Or, dans le cas de l'adaptation du Mangeur d'opium, on se rend compte que son rôle est essentiel, puisqu'elle est confrontation, obligation au texte et à l'œuvre à traduire, et l'exercice de la traduction est justement celui qui permet à Baudelaire de faire l'expérience de De Quincey et de la dépasser. Un mangeur d'opium mobilise les questionnements essentiellement métaphysiques et esthétiques de Baudelaire. Dans son rapport à l'écriture, un raisonnement et une théorie se déploient, se démontrent et s'expérimentent. Il ne faut pas négliger la portée d'Un mangeur d'opium. Plus qu'une simple adaptation, c'est un hymne à la création artistique, un vibrant hommage à un auteur aimé, l'affirmation d'une position 
éthique grandiose, qui se construit dans l'espoir d'un monde qui verrait le règne de la Beauté en quoi se réaliserait la communion de la communauté des êtres souffrants.

Baudelaire est un poète qui réussit à faire d'une rencontre littéraire exemplaire une expérience esthétique essentielle; à intégrer sa parole à celle d'autrui sans la dénaturer. L'on peut donc dire que Baudelaire - à la manière de l'auteur anglais «mangeur d'opium» - est «mangeur des Confessions»: il absorbe l'œuvre tout en la révélant.

\section{Références}

Bastin, Georges L. (1993). «La notion d'adaptation en traduction». Meta, 38, 3, p. 473-478.

Baudelaire, Charles (1973a). Correspondance I: 1832-1860. Texte établi, présenté et annoté par Claude Pichois avec la collaboration de Jean Ziegler. Paris, Gallimard, Bibliothèque de la Pléiade.

Baudelaire, Charles (1973b). Correspondance II: 1860-1866. Texte établi, présenté et annoté par Claude Pichois avec la collaboration de Jean Ziegler. Paris, Gallimard, Bibliothèque de la Pléiade.

Baudelaire, Charles (1975). Euvres complètes, tome I. Texte établi, présenté et annoté par Claude Pichois. Paris, Gallimard, Bibliothèque de la Pléiade.

Baudelaire, Charles (1976). Euvres complètes, tome II. Texte établi, présenté et annoté par Claude Pichois, Paris, Gallimard, Bibliothèque de la Pléiade.

Bonnefoy, Yves (2000). Baudelaire: la tentation de l'oubli. Paris, Bibliothèque Nationale de France.

Butor, Michel (1992). Essai sur les Modernes. Paris, Gallimard.

Dayre, Éric (2003). «Portrait du mangeur d'opium en prosateur et théoricien romantique». In Laurent Bury, dir. Confessions of an English Opium-Eater. Ellipses, p. 180-190.

Dayre, Éric (2009). L'Absolu comparé. Paris, Hermann.

De Quincey, Thomas (1998 [1821]). Confessions of an English OpiumEater, Oxford, Oxford University Press.

De Quincey, Thomas (2000 [1821]). Les Confessions d'un mangeur d'opium anglais. Trad. Pierre Leyris. Paris, Gallimard.

Gambier, Yves (1992). «Adaptation: une ambiguïté à interroger». Meta, 37, 3, p. 421-425.

Jakobson, Roman (1959). "On Linguistic Aspects of Translation». In R. Brower, dir. On Translation. Cambridge (MA), Harvard University Press, p. 233. 
Johnson, M. A. (1984). «Translation and Adaptation». Meta, 29, 4, p. $421-425$.

Milner, Max (1996). «Le Paradis se gagne-t-il?». Année Baudelaire 2, Klincksieck, p. 11-24.

Milner, Max (2000). L'Imaginaire des drogues, de Thomas De Quincey à Henri Michaux. Paris, Gallimard.

Pichois, Claude (1967). Lettres à Charles Baudelaire, Études Baudelairiennes. Neuchâtel, La Baconnière.

Pichois, Claude (1975). «Notice». In Charles Baudelaire, Euvres complètes, tome I. Paris, Gallimard, Bibliothèque de la Pléiade, p. 788-1606.

Scott, Clive (2006). Translating Rimbaud's Illuminations. Exeter, University of Exeter Press.

Camille Muris-Prime Ecole Jeannine Manuel, 43-45 Bedford Square WC1B 3DN London UNITED KINGDOM 